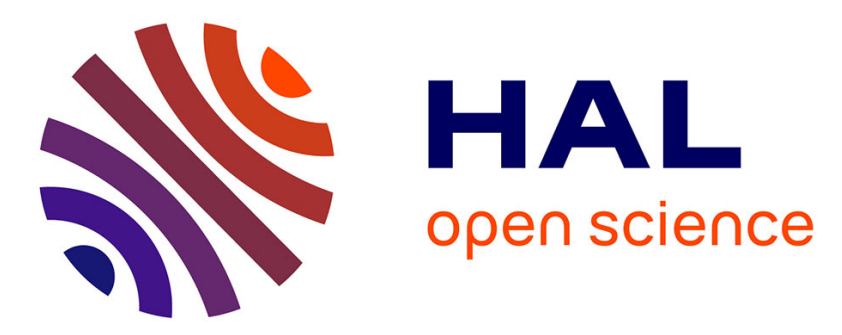

\title{
TCE+: An Extension of the TCE Method for Detecting Equivalent Mutants in Java Programs
}

\author{
Mahdi Houshmand, Samad Paydar
}

\section{To cite this version:}

Mahdi Houshmand, Samad Paydar. TCE+: An Extension of the TCE Method for Detecting Equivalent Mutants in Java Programs. 7th International Conference on Fundamentals of Software Engineering (FSEN), Apr 2017, Teheran, Iran. pp.164-179, 10.1007/978-3-319-68972-2_11 . hal-01760849

\section{HAL Id: hal-01760849 \\ https://hal.inria.fr/hal-01760849}

Submitted on 6 Apr 2018

HAL is a multi-disciplinary open access archive for the deposit and dissemination of scientific research documents, whether they are published or not. The documents may come from teaching and research institutions in France or abroad, or from public or private research centers.
L'archive ouverte pluridisciplinaire HAL, est destinée au dépôt et à la diffusion de documents scientifiques de niveau recherche, publiés ou non, émanant des établissements d'enseignement et de recherche français ou étrangers, des laboratoires publics ou privés. 


\title{
TCE+: an Extension of the TCE Method for Detecting Equivalent Mutants in Java Programs
}

\author{
Mahdi Houshmand, Samad Paydar \\ Dependable Distributed Embedded Systems (DDEmS) Laboratory \\ Computer Engineering Dept., Ferdowsi University of Mashhad \\ Mashhad, Iran \\ mahdi.houshmandemail.um.ac.ir, s-paydareum.ac.ir
}

\begin{abstract}
While mutation testing is considered to be an effective technique in software testing, there are some impediments to its widespread use in industrial projects. One of these challenges is the equivalent mutant problem, and a line of research is dedicated to proposing new methods for addressing this problem. Trivial Compiler Equivalence (TCE) method is recently introduced as a simple technique that actually relies only on the optimizations made by the compiler. It is shown by empirical studies that employing TCE with the gcc compiler results in a fast and effective technique for detecting equivalent mutants in $C$ programs. However, considering the fact that the Java compilers generally do not perform noticeable optimizations, the question is how effectively does TCE perform on Java programs? In this paper, experimental evaluations are discussed which demonstrate that using TCE technique with javac compiler results in very poor performance. As a result, this paper proposes to use the Java obfuscators as the complementary component, because of the optimizations they make. The experimental evaluations confirm that using TCE with the ProGuard obfuscation tool provides an effective and efficient method for detecting equivalent mutants in Java programs.
\end{abstract}

Keywords: mutation testing; equivalent mutant; trivial compiler equivalence; Java.

\section{$1 \quad$ Introduction}

Mutation testing is considered to be an effective approach to evaluate and also to improve an existing test set [1]. It works based on the notion of mutants, where each mutant is created by making a simple modification on the program under test. The set of possible modifications are defined by the mutation operators that are defined for the programming language of the target program. If there is a test set that the program has successfully executed on, then mutation testing can be applied to provide a measure of the quality of that test set. This is performed by running each mutant $\mathrm{M}$ on the test cases to investigate whether the test cases are powerful enough to detect the injected fault, i.e. the mutation. If the result of running the mutant on a test cases is different from the 
result of running the original program on that test case, then the test case has been able to distinguish, or kill, that mutant. The greater ratio of the mutants of the program are killed by the test set, the higher is the score of that test set. Finally, if there remains any live mutant, i.e. mutants that are not killed by any test case, then there are two possible cases for each live mutant: 1) whether this is a sign of the weakness of the test set, or 2) the mutant is an equivalent mutant, i.e. the corresponding mutation has made a syntax change without changing the semantic, and hence, the mutant cannot be killed by any test case.

When applying mutation testing, a method is necessary to distinguish which of the above cases holds for a live mutant. Without differentiating these two cases, it is possible that the test case designer wastes his time and effort in trying to find a test case for killing an equivalent mutant, which is actually not killable. Further, an equivalent mutant may cause the quality of the test set to be underestimated.

While mutation testing has been empirically proven to be able to simulate real-world programming errors [24], and hence to be an effective method for evaluating and improving test sets, there some non-negligible impediments towards its application in industrial software. The first problem is that mutation testing is a costly method, since the number of possible mutants, even for a relatively small program is usually high. Creating the mutants, compiling and executing them over the test cases and comparing the execution result usually requires noticeable time and computation resources.

Another problem is the equivalent mutants introduced before. Consequently, different approaches have been introduced during the last two decades for addressing this problem by employing different techniques like machine learning [14], logical constraint solving [15], data flow pattern analysis [8], gamification [17], program slicing [10] and code similarity measures [13]. One of the approached introduced recently, is the Trivial Compiler Equivalence (TCE) approach [12] which is a simple, fast and effective technique for detecting equivalent mutants.

The TCE technique actually relies on the optimizations performed by the compiler, and it tries to determine equivalence of a mutant by comparing it with the original program, in their binary, i.e. compiled, format. TCE has been evaluated in [12] on C programs using the $g c c$ compiler that is capable of performing different levels of optimizations when compiling the program. The evaluations have shown that TCE is an effective method for equivalent mutant detection in C programs. Considering Java programs, however, TCE is not expected to perform noticeably, since the Java compiler performs almost no specific optimization, and it leaves the optimizations to be performed by Java Virtual Machine at runtime (JVM) [26]. We believe there is room for evaluating the TCE technique on Java programs. Hence, in this paper, we experimentally evaluate performance of TCE on Java programs, and further, we introduce TCE+ as an extension of TCE which utilizes the ProGuard ${ }^{1}$ Java obfuscator in addition to the compiler to address the lack of compiler optimizations.

The rest of the paper is organized as follows. Section 2 briefly reviews the related works. In Section 3, the experimental evaluation of the TCE and TCE+ techniques on Java programs is discussed. Finally, Section 4 concludes the paper.

\footnotetext{
${ }^{1} \mathrm{http}: / /$ proguard.sourceforge.net/
} 
TCE+: an Extension of the TCE Method for Detecting Equivalent Mutants in Java Programs

\section{Related Work}

In order to address the equivalent mutant problem in the mutation testing domain, different approaches have been proposed during the last two decades. This problem, in its general form is an undecidable problem $[2,3]$ and therefore it is not expected to be able to find an automated method that can solve every instance of this problem correctly and completely. As a result, some of the proposed approaches employ heuristics or limit the characteristics of the program under study, for instance restricting the number of iterations of the loops [25]. A literature review on the approaches for tackling with the equivalent mutant problem is provided in [4], where it is concluded that the equivalent mutant detection techniques are still "far from perfect".

Some works attempt to deterministically determine whether a specific mutant is equivalent or not. For instance, in $[8,18]$ a set of 9 data flow patterns is introduced that result in equivalent mutants. In addition, a framework is proposed which uses static analysis of data flow to check each mutant of a program against these patterns. If a mutant follows one of the predefined patterns, then it is equivalent, otherwise it is considered to be non-equivalent. As another example, [15] introduces a technique that extracts a set of logical constraints from a mutant such that solving those constraints proves that the mutant is equivalent to the original program. Then, the constraints are given to a constraint solver tool for the purpose of detecting equivalent mutants. The method assumes certain characteristics on the mutants which limits applicability of the method (e.g. recursive functions are not supported). A similar approach based on constraint solving techniques is also introduced in [16].

Some works implicitly use the idea that for an undecidable problem, it is not possible to provide a complete automated solution and hence human intervention is unavoidable. Therefore, they try to help the human experts in analyzing the mutants and in making decision about their equivalence. This help can be provided in form of identifying the mutants that are more likely to be equivalent. Therefore, these methods follow a inexact approach and generate a recommended list of mutants, ordered by their equivalence probability, that need to be manually analyzed by the human expert to make the final decision. For instance, in [11], the idea is that the probability that a mutant is not equivalent is related to how its coverage on a specific test set differs from the coverage of the original program. In other words, the greater the coverage is affected, the lower is the probability of the mutant being equivalent. A similar approach for determining equivalent mutants based on the coverage impact is also proposed in [6, 5]. Machine learning techniques are also used in some works like [14] to provide a probabilistic approach to detection of equivalent mutants.

Another example of the works that count on human involvement for detection of equivalent mutants is [17] that uses gamification technique. It introduces a two-player game in which one player tries to create mutants that are hard to kill, and the other one tries to introduce test cases that kill the mutants. The game indirectly can contribute to detecting mutants that are more likely to be equivalent.

Another group of works try to avoid creation of equivalent mutants by more advanced mutation generation techniques. For instance, [19] proposes to consider the fact 
that different mutation operators perform differently from the point of view of the difficulty of killing their resulting mutants. This can be employed to selectively use mutation operators that less frequently create equivalent mutants. Another group of works have shown that using higher order mutants instead of first-order mutants can reduce the number of equivalent mutants generated for a program $[9,20,21,22]$.

Other techniques that have been used for exact equivalent mutant detection include code similarity measures and clone detection techniques [13], program slicing techniques [10], co-evolution algorithms [7].

An interesting approach that is recently proposed for detection of the equivalent mutants is the TCE approach [12], which uses a very simple and straightforward technique. TCE works based on the idea that the advanced optimizations performed by a compiler can remove some type of the mutations that have not affected the semantic of the program, and hence if the equivalent mutant is compiled, the result of compiling can be the same as the result of compiling the original program. It is demonstrated through experimental evaluations that the TCE technique is successful in effectively detecting equivalent mutants of a $\mathrm{C}$ program using the $g c c$ compiler optimizations. However, since the Java compilers generally do not perform noticeable optimizations, the performance of TCE on Java programs needs to be investigated. As a result, current paper proposes TCE+ technique as an extension of TCE that utilizes ProGuard for the purpose of optimizing Java code. In addition to performing different optimizations, e.g. dead code removal, unused variable removal and peephole optimizations, ProGuard is also able to obfuscate, shrink and pre-verify Java byte codes. However, TCE+ uses ProGuard only for the purpose of optimizations and it does not use obfuscation or shrinking capabilities of ProGuard. It is beyond the scope of this paper to describe the optimization techniques employed by ProGuard or $g c c$, however, Table $\mathbf{1}$ briefly mentions some of the main optimizations performed by each of these tools.

In [12], TCE has been shown to be able to find, in addition to equivalent mutants, the duplicated mutants, i.e. mutants that are equivalent to each other, but not necessarily equivalent to the original program. Since there is no advantage in using two duplicated mutants, it is interesting to be able to detect duplicated mutants. In this paper, we evaluate the TCE and TCE+ methods for the purpose of detecting equivalent and duplicated mutants of Java programs.

Table 1. Some of the optmization techniques employed by the subject tools

\begin{tabular}{|c|l|}
\hline Tool & \multicolumn{1}{|c|}{ Optimization Techniques } \\
\hline gcc Compiler & $\begin{array}{l}\text { Dead Code Elimination, Transforming Conditional Jumps, Constant Folding, De- } \\
\text { Virtualization, Function Inlining, Predictive Commoning, Elimination of Useless } \\
\text { Null Pointer Checks, Peephole Optimization, Global Common Subexpression Elimi- } \\
\text { nation }\end{array}$ \\
\hline ProGuard & $\begin{array}{l}\text { Dead Code Elimination, Peephole Optimization, Marking Classes as Final, } \\
\text { Variable Allocation Optimization, Method Inlining, Return Value Propagation, Re- } \\
\text { moving Write-only Fields }\end{array}$ \\
\hline
\end{tabular}


TCE+: an Extension of the TCE Method for Detecting Equivalent Mutants in Java Programs

\section{Experimental Study}

In this section, the experimental evaluation of the TCE and TCE+ approaches over Java programs is discussed. First, the research questions are introduced and then, different elements of the experiments are described. Finally, the results of the experiments are discussed.

\subsection{Research Questions}

Since the TCE approach has been shown to be both effective and efficient in detecting equivalent and duplicated mutants in $\mathrm{C}$ programs, the main research question this paper seeks to answer is:

RQ. How do the TCE and TCE+ approaches perform on Java programs?

To answer this question, two more specific research questions are introduced:

RQ1. How effective are the TCE and TCE+ approaches at detecting equivalent and duplicated mutants in Java programs?

To answer this question, the number of equivalent and duplicated mutants detected by the TCE and TCE+ techniques, and also the ratio of the detected equivalent mutants to the existing equivalent mutants is reported.

RQ2. How efficient is TCE+ for the purpose of equivalent mutant detection?

This question is answered by computing the execution time of the TCE+ approach to see if it is efficient enough to be used in practice. While we have not evaluated TCE+ on large programs, we believe that the efficiency of the technique for the large programs can be estimated based on the results obtained for the small programs.

\subsection{Dataset and Golden Standard}

For the purpose of the experimental evaluations, first, a dataset is prepared including 5 java programs, and then, for each program, its mutants are created by the MuJava mutation testing tool [23]. Table 2 shows the name of each program, its size in terms of physical Source Line of Code (SLOC) and the number of its mutants. The mutation operators that MuJava has applied on the subject programs are mentioned in Table 3.

In addition, a golden standard is created by manually checking each mutant of the subject programs to determine whether it is equivalent to the original program. This manual analysis is performed separately by three experts who have had more than 10 years of experience in object oriented programming in Java. After each expert has finished his job, the results have been compared so that any possible conflict is resolved. Actually, there were 7 such cases that needed the experts to discuss with each other to agree on the result. 
Table 2. Dataset used in the experiments

\begin{tabular}{|c|l|c|c|}
\hline Program & \multicolumn{1}{|c|}{ Subject Program } & Physical SLOC & Number of Mutants \\
\hline P1 & BubbleSort & 15 & 111 \\
\hline P2 & Bisect & 25 & 189 \\
\hline P3 & Triangle & 46 & 456 \\
\hline P4 & QuickSort & 50 & 341 \\
\hline P5 & java.util.StringTokenizer & 174 & 772 \\
\hline
\end{tabular}

Table 3. Mutation operators applied by MuJava on the subject programs

\begin{tabular}{|c|c|}
\hline Operator & Operator Definition \\
\hline AODS: Short-cut Arithmetic Operator Deletion & $\{(x, \operatorname{remove}(x)) \mid x \in\{++,--\}\}$ \\
\hline AODU: Unary Arithmetic Operator Deletion & $\{(-\mathrm{v}, \mathrm{v})\}$ \\
\hline AOIS: Short-cut Arithmetic Operator Insertion & $\{(\mathrm{v},--\mathrm{v}),(\mathrm{v}, \mathrm{v}--),(\mathrm{v},++\mathrm{v}),(\mathrm{v}, \mathrm{v}++)\}$ \\
\hline AOIU: Unary Arithmetic Operator Insertion & $\{(\mathrm{v},-\mathrm{v})\}$ \\
\hline AORB: Binary Arithmetic Operator Replacement & $\left\{(\mathrm{x}, \mathrm{y}) \mid \mathrm{x}, \mathrm{y} \in\left\{+,-,{ }^{*}, /, \%\right\} \wedge \mathrm{x} \neq \mathrm{y}\right\}$ \\
\hline AORS: Shortcut Arithmetic Operator Replacement & $\{(\mathrm{x}, \mathrm{y}) \mid \mathrm{x}, \mathrm{y} \in\{++,--\} \wedge \mathrm{x} \neq \mathrm{y}\}$ \\
\hline ASRS: Shortcut Assignment Operator Replacement & $\{(\mathrm{x}, \mathrm{y}) \mid \mathrm{x}, \mathrm{y} \in\{+=,-=, *=, /=, \%=\} \wedge \mathrm{x} \neq \mathrm{y}\}$ \\
\hline CDL: Constant DeLetion & $\begin{array}{l}\{(\text { op c, remove }(\text { op c })) \mid \text { op } \in\{+,-, *, /, \%,>, \\
>=,<,<=\}\}\end{array}$ \\
\hline COD: Conditional Operator Deletion & $\{(!(\mathrm{e}), \mathrm{e}) \mid \mathrm{e} \in\{$ if(e), while $(\mathrm{e})$, for $(\mathrm{s} ; \mathrm{e} ; \mathrm{s})\}\}$ \\
\hline COI: Conditional Operator Insertion & $\{(\mathrm{e}, !(\mathrm{e})) \mid \mathrm{e} \in\{$ if $(\mathrm{e})$, while $(\mathrm{e})$, for $(\mathrm{s} ; \mathrm{e} ; \mathrm{s})\}\}$ \\
\hline COR: Conditional Operator Replacement & $\{(\mathrm{x}, \mathrm{y}) \mid \mathrm{x}, \mathrm{y} \in\{\& \&, \|, \wedge\} \wedge \mathrm{x} \neq \mathrm{y}\}$ \\
\hline LOI: Logical Operator Insertion & $\{(\mathrm{v}, \sim \mathrm{v})\}$ \\
\hline ODL: Operator DeLetion & $\begin{array}{l}\{(\mathrm{v} \text { op, } \operatorname{remove}(\mathrm{v} \text { op })),(\text { op v, } \operatorname{remove}(\text { op v })) \mid \\
\text { op } \in\{+,-, *, /, \%,<,<=,>,>=\}\},\{(\mathrm{v}++, \mathrm{v}), \\
(\mathrm{v}--, \mathrm{v}),(--\mathrm{v}, \mathrm{v}),(++\mathrm{v}, \mathrm{v}) \mid \text { op } \in\{++,--\}\}\end{array}$ \\
\hline ROR: Relational Operator Replacement & $\{(\mathrm{x}, \mathrm{y}) \mid \mathrm{x}, \mathrm{y} \in\{>,>=,<,<=,==, !=\} \wedge \mathrm{x} \neq \mathrm{y}\}$ \\
\hline SDL: Statement DeLetion & $\{(\mathrm{s}$, remove $(\mathrm{s}))\}$ \\
\hline VDL: Variable DeLetion & $\begin{array}{l}\{(\mathrm{v}[\mathrm{op}], \operatorname{remove}(\mathrm{v}[\mathrm{op}])) \mid \text { op } \in\{+,-, *, /, \%, \\
++,--,<,<=,>,>=\}\end{array}$ \\
\hline
\end{tabular}

\subsection{Experimental Environment}

All the experiments are performed on a PC with Microsoft Windows 7 operating system, Intel Core i5-4400 processor and 8GB RAM. Further, we have used the Oracle's Java compiler javac version 1.8.0_60 to compile the programs and the mutants, and also ProGuard 5.3 to optimize the compilation results. Finally, for the purpose of comparing the binary files, the Windows utility program FC is used with the parameters $/ \mathrm{B}$ and /LB1.

\subsection{Experiments}

To answer the research questions, four experiments are designed. The first two experiments evaluate the TCE and TCE+ techniques for the purpose of equivalent mutant detection and the second two experiments evaluate them for detecting duplicated mutants. The processes used in these experiments are shown in Fig. 1 to Fig. 4. 
TCE+: an Extension of the TCE Method for Detecting Equivalent Mutants in Java Programs

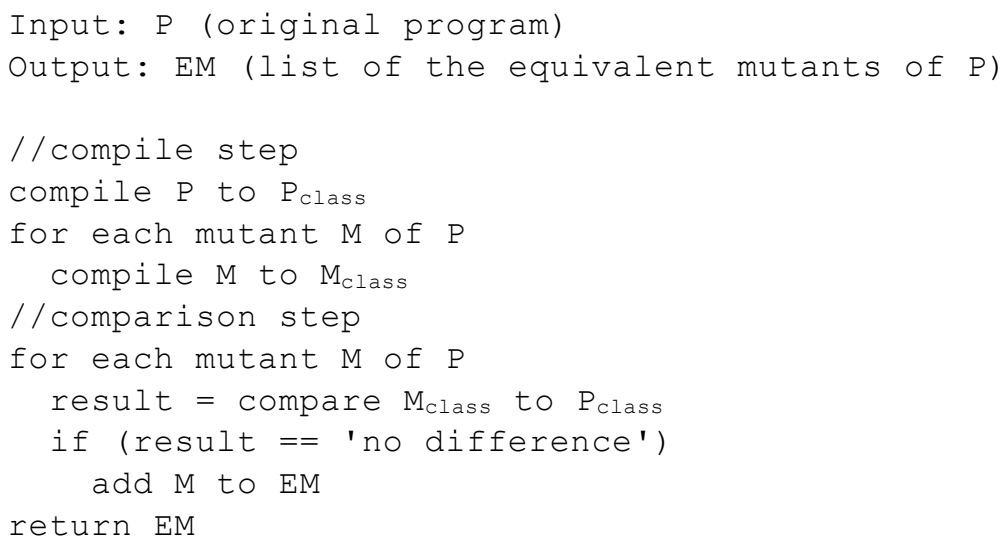

Fig. 1. Process of experiment 1: TCE for equivalent mutant detection

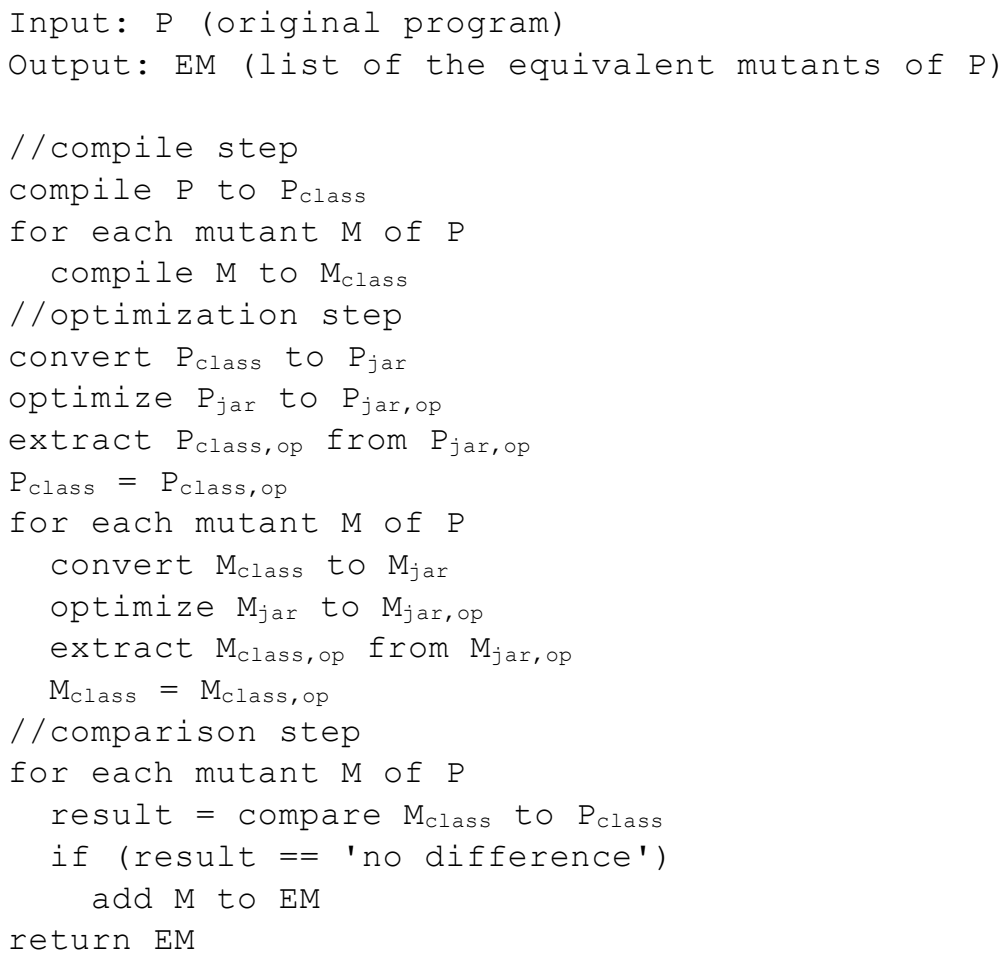

Fig. 2. Process of experiment 2: TCE+ for equivalent mutant detection 
In the first experiment, for each subject program $\mathrm{P}, \mathrm{P}$ is compiled to $\mathrm{P}_{\text {class }}$ and each mutant $\mathrm{M}$ of $\mathrm{P}$ is compiled to $\mathrm{M}_{\text {class. }}$. Then each compiled mutant $\mathbf{M}_{\text {class }}$ is compared to the $\mathrm{P}_{\text {class. }}$ If no difference is identified in this comparison, it is considered that TCE has determined the corresponding mutant as an equivalent mutant.

The second experiment evaluates the TCE+ approach by including an optimization phase before the comparison step. In order to perform the optimization, first a jar file is created from the compiled file, i.e. $\mathrm{P}_{\text {class }}$ or $\mathrm{M}_{\text {class. }}$ The jar file is then given to ProGuard to do the optimizations. The resulting jar file is then decompressed to extract the optimized compiled file which then goes through the binary comparison.

In the third experiment, each compiled mutant of the program is compared to all other compiled mutants of that program that have the same file size. If there is no difference between the corresponding binary files, those two mutants are added as a pair to the list of duplicated mutants. After processing all the mutants, a simple algorithm shown in Fig. 3 is used to determine the list of mutants that can be removed.

The fourth experiment is very similar to the third experiment and the only difference is that it compares the optimized version of the compiled mutants which are created by the process described for the second experiment.

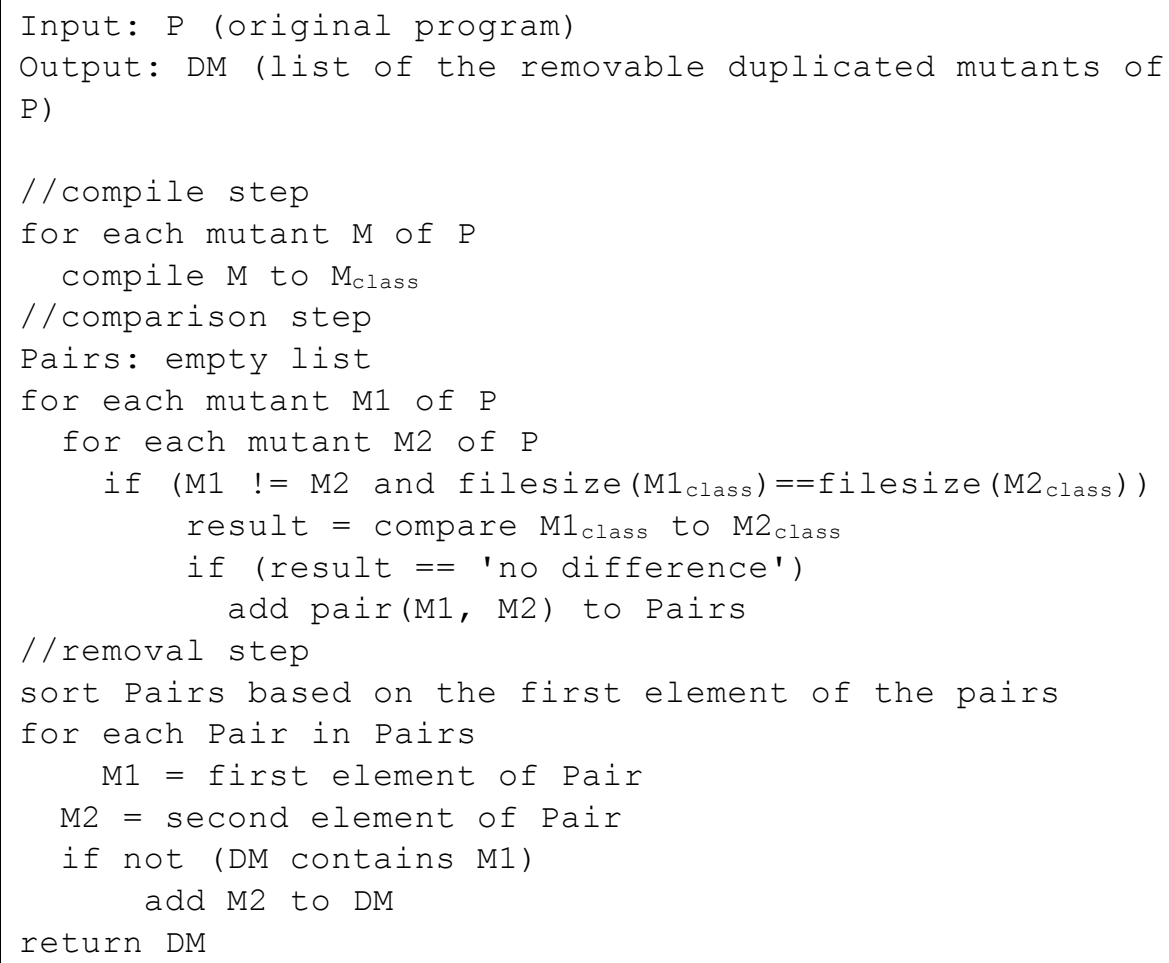

Fig. 3. Process of experiment 3: TCE for duplicated mutant detection 
TCE+: an Extension of the TCE Method for Detecting Equivalent Mutants in Java Programs

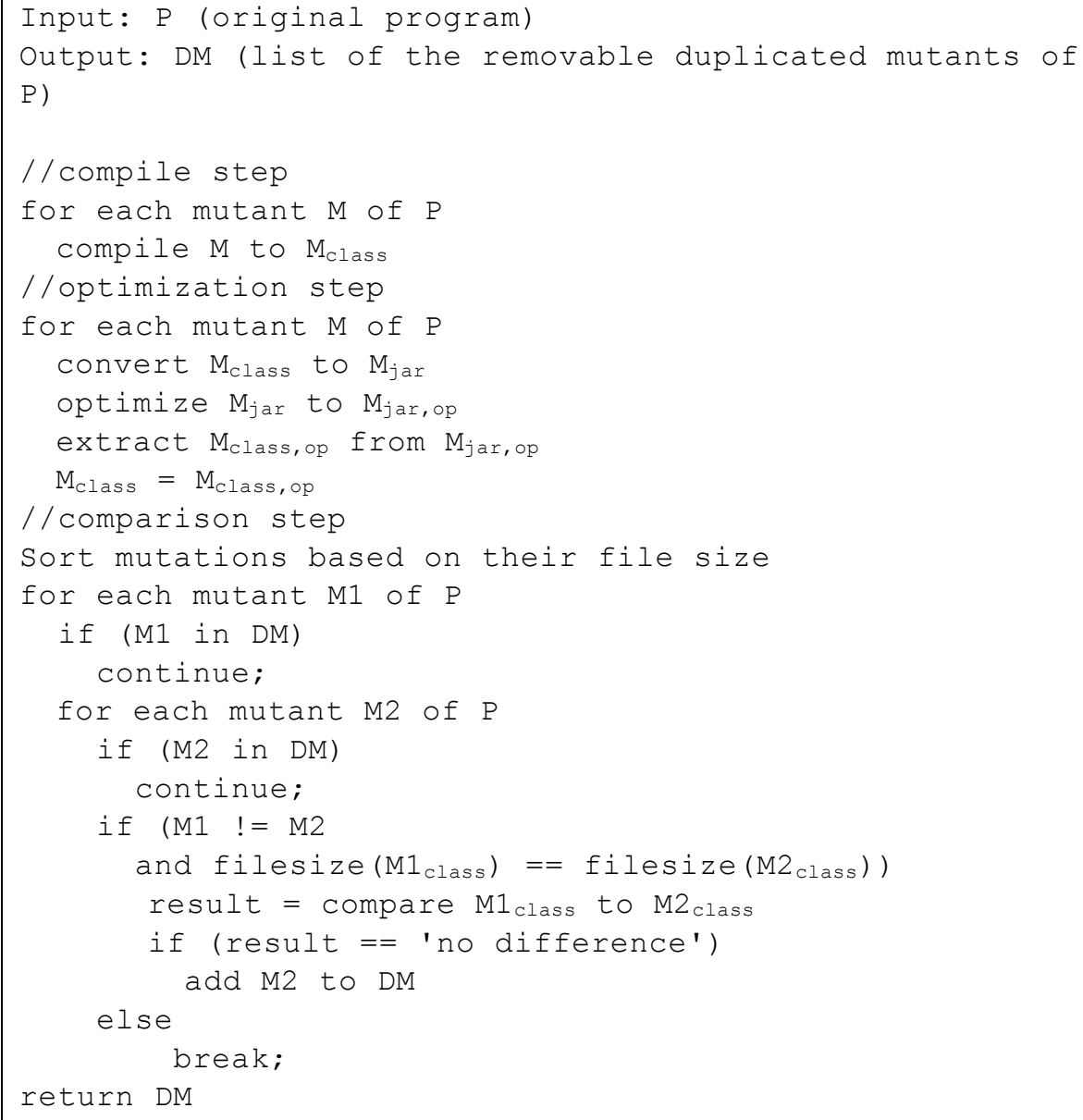

Fig. 4. Process of experiment 4: TCE+ for duplicated mutant detection

\subsection{Result Analysis}

The results of the first two experiments are shown in Table 4. As it is shown in this table, TCE approach has not detected any equivalent mutant in the subject programs. Therefore, it can be concluded that since the Java compiler does not perform noticeable optimizations [26], applying TCE on Java programs is not effective for detecting equivalent mutants. However, the TCE+ technique, which compensates the limitation of the Java compiler by utilizing ProGuard's optimizations, has identified some equivalent mutants for each of the subject programs. Therefore, TCE+ has been able to address the shortcomings of the TCE method. However, the number of detected equivalent mutants is small and at the best case, i.e. the Bisect program, it accounts for only $7 \%$ of all 
the mutants. The worst case is also the BubbleSort program that the detected equivalent mutants are only $2 \%$ of all the mutants.

In order to judge the effectiveness of the TCE+ approach, it is required to know the ratio of the detected equivalent mutants to all the existing equivalent mutants. Therefore, the results of the first two experiments have been compared with the golden standard. As shown in the last column of Table 4, TCE+ has been able to detect from 18\% to $100 \%$ of all the existing equivalent mutants. It has missed 9, 2 and 7 equivalent mutants respectively for the BubbleSort, QuickSort and StringTokenizer programs. For the other two programs, i.e. Bisect and Triangle, all the existing equivalent mutants have been found by TCE+.

Based on these results, we conclude that TCE+ is generally effective and it is successful in detecting a good ratio of the existing equivalent mutants. However, it is interesting to analyze the detected and undetected equivalent mutants based on their mutation operators.

The distribution of the mutation operators over all the generated mutants is shown in Table 5. The top-3 mutation operators that have created the greatest proportion of the mutants are AOIS, ROR and SDL, which have created respectively 33\%, 20\% and $10 \%$ of all the mutants. There are some operators like AOSE and AODU that have negligible contribution to the number of mutants created.

Table 4. Results of Experiments 1 and 2: Detecting Equivalent Mutants

\begin{tabular}{|c|c|c|c|c|c|c|}
\hline \multirow{2}{*}{ Program } & \multicolumn{2}{|c|}{$\begin{array}{l}\text { Number of Detected } \\
\text { Equivalent Mutants }\end{array}$} & \multicolumn{2}{|c|}{$\begin{array}{c}\text { Percentage of Detected } \\
\text { Equivalent Mutants to All } \\
\text { Mutants }\end{array}$} & \multicolumn{2}{|c|}{$\begin{array}{c}\text { Percentage of Detected Equiva- } \\
\text { lent Mutants to All Existing } \\
\text { Equivalent Mutants }\end{array}$} \\
\cline { 2 - 7 } & TCE & TCE+ & TCE & TCE+ & TCE & TCE+ \\
\hline P1 & 0 & 2 & 0 & 2 & 0 & 18 \\
\hline P2 & 0 & 14 & 0 & 7 & 0 & 100 \\
\hline P3 & 0 & 23 & 0 & 5 & 0 & 100 \\
\hline P4 & 0 & 10 & 0 & 3 & 0 & 83 \\
\hline P5 & 0 & 34 & 0 & 4 & 0 & 83 \\
\hline
\end{tabular}

Table 5. Distribution of the Mutation Operators Over All the Mutants

\begin{tabular}{|c|c|c|c|c|c|c|c|c|c|c|c|c|c|c|c|c|}
\hline \multirow[b]{2}{*}{ Program } & \multicolumn{16}{|c|}{ Mutation Operator } \\
\hline & Oิ & $\stackrel{2}{0}$ & $\stackrel{\mathscr{O}}{\alpha}$ & $\underset{2}{2}$ & 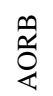 & 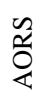 & $\frac{\tilde{\sigma}}{\tilde{\psi}}$ & $\overrightarrow{\mathrm{\partial}}$ & ठิ & $\overline{0}$ & 号 & ఠ] & $\overrightarrow{0}$ & $\begin{array}{l}\stackrel{0}{0} \\
\simeq\end{array}$ & $\overrightarrow{\hat{n}}$ & $\vec{\rho}$ \\
\hline P1 & & & 30 & 3 & 16 & 2 & & 4 & & 3 & & 11 & 8 & 19 & 10 & 5 \\
\hline $\mathrm{P} 2$ & & & 80 & 13 & 32 & & & 2 & & 3 & & & 16 & 19 & 14 & 10 \\
\hline P3 & & & 128 & 11 & 36 & & & 3 & & 24 & 14 & 43 & 32 & 119 & 31 & 15 \\
\hline $\mathrm{P} 4$ & 2 & & 108 & 18 & 36 & 6 & & 8 & & 9 & & 40 & 20 & 55 & 28 & 11 \\
\hline P5 & & 2 & 262 & 33 & & 7 & 20 & & 6 & 39 & 20 & 80 & 33 & 163 & 100 & 7 \\
\hline Total & 2 & 2 & 608 & 78 & 120 & 15 & 20 & 17 & 6 & 78 & 34 & 174 & 109 & 375 & 183 & 48 \\
\hline $\operatorname{Ratio}_{(\%)^{1}}$ & $<1$ & $<1$ & 33 & 4 & 6 & 1 & 1 & 1 & $<1$ & 4 & 2 & 9 & 6 & 20 & 10 & 3 \\
\hline
\end{tabular}

${ }^{1}$ percentage to all the mutants 
TCE+: an Extension of the TCE Method for Detecting Equivalent Mutants in Java Programs

In Table 6, the distribution of the mutation operators over all the existing equivalent mutants is shown. An interesting point is that the AOIS operator which has created about $33 \%$ of all the mutants is also responsible for creating about $77 \%$ of all the equivalent mutants in the golden standard. Further, the ROR operator has created about $14 \%$ of all the equivalent mutants. From another point of view, about $13 \%$ of the mutants created by the AOIS operator have been equivalent. This value for the ROR operator has been about $4 \%$. This means that the performance of the TCE+ technique over these two mutation operators is of greater importance, compared to other mutation operators.

The distribution of the mutation operators over all the equivalent mutants that are found by TCE+ is shown in Table 7. Comparing this table with Table 6 shows that TCE+ has successfully detected all the equivalent mutants created by the AOIS operator, which account for about $77 \%$ of all the equivalent mutants. Hence, considering the ratio of AOIS-generated equivalent mutants, it can be concluded that the TCE+ approach is an effective method for detection of equivalent mutants in Java programs. However, it is also important to note that TCE+ has not detected any of the 14 equivalent mutants created by the ROR operator ( 5 for BubbleSort, 2 for QuickSort and 7 for StringTokenizer). It also has missed 4 other equivalent mutants of BubbleSort, 2 created by the AORB operator, 1 by ODL and 1 by the CDL operator.

Regarding detection of the duplicated mutants, the results of the third and the fourth experiments are presented in Table 8. This table shows that TCE and TCE+ have identified respectively from $8 \%$ to $14 \%$ and from $13 \%$ to $23 \%$ of the mutants of the subject programs as being duplicated. Since the duplicated mutants do not contribute to the mutation testing results, they can be removed from the mutants. Considering all the five subject programs, TCE and TCE+ have identified respectively $9 \%$ and $16 \%$ of all the mutants as being duplicated. As a result, we conclude that while TCE+ noticeably outperforms TCE, both approaches are effective in detecting duplicated mutants.

Table 6. Distribution of the Mutation Operators Over the Existing Equivalent Mutants

\begin{tabular}{|c|c|c|c|c|c|c|c|c|c|c|c|c|c|c|c|c|}
\hline \multirow[b]{2}{*}{ Program } & \multicolumn{16}{|c|}{ Mutation Operator } \\
\hline & $\frac{2}{2}$ & $\stackrel{?}{2}$ & 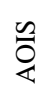 & $\stackrel{\vartheta}{\varrho}$ & $\frac{n}{2}$ & $\frac{\mathscr{1}}{0}$ & $\begin{array}{l}\tilde{\alpha} \\
\frac{\tilde{w}}{\alpha}\end{array}$ & $\vec{\vartheta}$ & Оิ & $\overline{0}$ & 苞 & రెత & $\overrightarrow{0}$ & $\stackrel{\Upsilon}{0}$ & $\overrightarrow{\hat{\sigma}}$ & 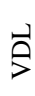 \\
\hline P1 & & & 2 & & 2 & & & 1 & & & & & 1 & 5 & & \\
\hline $\mathrm{P} 2$ & & & 12 & 2 & & & & & & & & & & & & \\
\hline P3 & & & 20 & 1 & & & & & & & & & 1 & & & 1 \\
\hline P4 & & & 10 & & & & & & & & & & & 2 & & \\
\hline P5 & & & 34 & & & & & & & & & & & 7 & & \\
\hline Total & 0 & 0 & 78 & 3 & 2 & 0 & 0 & 1 & 0 & 0 & 0 & 0 & 2 & 14 & 0 & 1 \\
\hline${\operatorname{Ratio}(\%)^{1}}^{1}$ & 0 & 0 & 77 & 3 & 2 & 0 & 0 & 1 & 0 & 0 & 0 & 0 & 2 & 14 & 0 & 1 \\
\hline
\end{tabular}

${ }^{1}$ Percentage to Existing Equivalent Mutants 
Table 7. Distribution of the Operators Over the Equivalent Mutants Detected by TCE+

\begin{tabular}{|c|c|c|c|c|c|c|c|c|c|c|c|c|c|c|c|c|}
\hline \multirow[b]{2}{*}{ Program } & \multicolumn{16}{|c|}{ Mutation Operator } \\
\hline & Oิ & 客 & $\stackrel{\Omega}{0}$ & 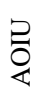 & $\frac{n}{2}$ & $\begin{array}{l}\text { है } \\
\text { Oै }\end{array}$ & 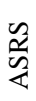 & $\vec{\theta}$ & Оิ & $\overline{0}$ & 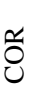 & $\widetilde{\sigma}^{\top}$ & $\overrightarrow{\widehat{\partial}}$ & 号 & $\overrightarrow{\hat{n}}$ & $\vec{\rho}$ \\
\hline P1 & & & 2 & & & & & & & & & & & & & \\
\hline $\mathrm{P} 2$ & & & 12 & 2 & & & & & & & & & & & & \\
\hline P3 & & & 20 & 1 & & & & & & & & & 1 & & & 1 \\
\hline P4 & & & 10 & & & & & & & & & & & & & \\
\hline P5 & & & 34 & & & & & & & & & & & & & \\
\hline Total & 0 & 0 & 78 & 3 & 0 & 0 & 0 & 0 & 0 & 0 & 0 & 0 & 1 & 0 & 0 & 1 \\
\hline Ratio (\%) ${ }^{1}$ & 0 & 0 & 94 & 4 & 0 & 0 & 0 & 0 & 0 & 0 & 0 & 0 & 1 & 0 & 0 & 1 \\
\hline
\end{tabular}

${ }^{1}$ Percentage to All Equivalent Mutants Detected by TCE+

Table 8. Results of Experiments 3 and 4: Detecting Duplicated Mutants

\begin{tabular}{|c|c|c|c|c|}
\hline \multirow{2}{*}{ Program } & \multicolumn{2}{|c|}{$\begin{array}{c}\text { umber of Detected Du- } \\
\text { plicated Mutants }\end{array}$} & $\begin{array}{r}\text { Percentage of Detected Duplicated Mu- } \\
\text { tants to All Mutants }\end{array}$ \\
\cline { 2 - 5 } & TCE & TCE+ & TCE & TCE+ \\
\hline P1 & 15 & 25 & 14 & 23 \\
\hline P2 & 16 & 31 & 8 & 16 \\
\hline P3 & 52 & 89 & 11 & 20 \\
\hline P4 & 34 & 59 & 10 & 17 \\
\hline P5 & 60 & 99 & 8 & 13 \\
\hline
\end{tabular}

An interesting point is that while TCE has not detected any equivalent mutant, but it has detected non-negligible number of duplicated mutants. Further analysis of the results reveals that the detected duplicated mutants are not a result of the optimizations made by TCE, but they are resulted from the fact that applying some MuJava mutation operators on some program statements may create exactly the same syntactic changes. In other words, for each pair of duplicated mutants detected by TCE, both mutants are syntactically-equal. An example pair is shown in Table 9. While TCE+ has detected all the duplicated mutants found by TCE, it has also detected other results which are syntactically different but semantically duplicated. An example is shown in Table $\mathbf{1 0 .}$

Another interesting point is that, as shown in Table 11, 44\% of all the duplicated mutants detected by TCE are created by the ROR operator. The other $23 \%$ are associated with the VDL operator. Only about $1 \%$ of the detected duplicated mutants are results of the AOIS operator. The results for the TCE+ technique are also presented in Table 12. This table shows that, compared to TCE, the TCE+ technique is able to detect the duplicated mutants that are created by a wider set of mutation operators. Actually, TCE+ has detected duplicated mutants of type AOI, AORB, CDL and LOI operators, of which none is detected by the TCE method.

Finally, to answer RQ1, we conclude that TCE is not effective for detecting equivalent mutants of Java programs, but it can effectively detect the duplicated mutants. Further, TCE+ is effective for detecting both equivalent and duplicated mutants. 
TCE+: an Extension of the TCE Method for Detecting Equivalent Mutants in Java Programs

Table 9. An Example Duplicated Mutant Detected by TCE

\begin{tabular}{|l|l|l|}
\hline Original Statement & Mutant by ODL Operator & Mutant by CDL Operator \\
\hline $\mathrm{x}=(\mathrm{M}+\mathrm{x}) / 2 ;$ & $\mathrm{x}=\mathrm{M}+\mathrm{x} ;$ & $\mathrm{x}=\mathrm{M}+\mathrm{x} ;$ \\
\hline
\end{tabular}

Table 10. An Example Duplicated Mutant Detected by TCE+ but Missed by TCE

\begin{tabular}{|c|l|l|}
\hline \multicolumn{1}{|c|}{ Original Statement } & Mutant by AOIS Operator & \multicolumn{1}{c|}{ Mutant by AOIS Operator } \\
\hline $\begin{array}{l}\text { public void setEpsilon(double } \\
\text { epsilon) }\{\end{array}$ & public void setEpsilon(double & public void setEpsilon(double \\
epsilon) \{ & epsilon) \{ \\
this.mEpsilon = epsilon; $\}$ & this.mEpsilon = epsilon--; $\}$ & this.mEpsilon = epsilon $++;\}$ \\
\hline
\end{tabular}

Table 11. Distribution of the Operators Over the Duplicated Mutants Detected by TCE

\begin{tabular}{|c|c|c|c|c|c|c|c|c|c|c|c|c|c|c|c|c|}
\hline \multirow[b]{2}{*}{ Program } & \multicolumn{16}{|c|}{ Mutation Operator } \\
\hline & $\frac{\tilde{O}}{2}$ & $\stackrel{\vartheta}{\stackrel{\vartheta}{\varrho}}$ & $\stackrel{2}{0}$ & 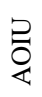 & $\frac{n}{2}$ & $\frac{\Omega}{0}$ & $\frac{n}{\tilde{\alpha}}$ & $\overrightarrow{\mathrm{\theta}}$ & Оి & ర & $\stackrel{\alpha}{0}$ & 어 & $\overrightarrow{0}$ & $\stackrel{\alpha}{0}$ & $\overrightarrow{\hat{\sigma}}$ & $\stackrel{\bar{\rho}}{\rho}$ \\
\hline $\mathrm{P} 1$ & & & & & & & & & & & & & 4 & 3 & 3 & 5 \\
\hline $\mathrm{P} 2$ & & & & & & & & & & & & & 4 & & 2 & 10 \\
\hline P3 & & & & & & & & & & & & & 3 & 27 & 7 & 15 \\
\hline $\mathrm{P} 4$ & & & 2 & & & & & & & & & & 10 & 9 & 5 & 8 \\
\hline P5 & & & & & & & & & & & & & 8 & 39 & 11 & 2 \\
\hline Total & 0 & 0 & 2 & 0 & 0 & 0 & 0 & 0 & 0 & 0 & 0 & 0 & 29 & 78 & 28 & 40 \\
\hline Ratio (\%) ${ }^{1}$ & 0 & 0 & 1 & 0 & 0 & 0 & 0 & 0 & 0 & 0 & 0 & 0 & 16 & 44 & 16 & 23 \\
\hline
\end{tabular}

${ }^{1}$ Percentage to All Duplicated Mutants Detected by TCE

Table 12. Distribution of the Operators Over the Duplicated Mutants Detected by TCE+

\begin{tabular}{|c|c|c|c|c|c|c|c|c|c|c|c|c|c|c|c|c|}
\hline \multirow[b]{2}{*}{ Program } & \multicolumn{16}{|c|}{ Mutation Operator } \\
\hline & $\stackrel{2}{0}$ & $\stackrel{2}{2}$ & $\stackrel{n}{0}$ & 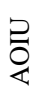 & $\frac{n}{2}$ & $\begin{array}{l}\mathscr{2} \\
\text { Oे }\end{array}$ & $\begin{array}{l}\tilde{\alpha} \\
\tilde{\sim} \\
\mathbb{4}\end{array}$ & $\overrightarrow{\mathrm{\theta}}$ & ○ิ & $\overline{0}$ & ○̂ & כ) & $\overrightarrow{0}$ & $\underset{\sim}{a}$ & $\overrightarrow{\hat{\sigma}}$ & $\stackrel{\bar{\rho}}{\rho}$ \\
\hline $\mathrm{P} 1$ & & & 1 & & 4 & & & 4 & & & & & 4 & 4 & 3 & 5 \\
\hline $\mathrm{P} 2$ & & & 13 & 2 & & & & & & & & & 4 & & 2 & 10 \\
\hline P3 & & & 19 & 1 & 3 & & & 1 & & & & 1 & 4 & 38 & 7 & 15 \\
\hline $\mathrm{P} 4$ & & & 14 & & 6 & & & 6 & & & & & 10 & 10 & 5 & 8 \\
\hline P5 & & & 34 & & & & & & & & & & 8 & 43 & 12 & 2 \\
\hline Total & 0 & 0 & 81 & 3 & 13 & 0 & 0 & 11 & 0 & 0 & 0 & 1 & 30 & 95 & 29 & 40 \\
\hline Ratio (\%) ${ }^{1}$ & 0 & 0 & 27 & 1 & 4 & 0 & 0 & 4 & 0 & 0 & 0 & 0 & 10 & 31 & 10 & 13 \\
\hline
\end{tabular}

${ }^{1}$ Percentage to All Duplicated Mutants Detected by TCE+

In order to evaluate efficiency of TCE+ for detecting equivalent mutants, its execution time for different steps, i.e. 1) compiling the mutants, 2) optimization of the compiled mutants, and 3) comparison of the optimization results, is separately measured for each subject program. The process of detecting duplicated mutants also includes the first two steps, but in the third step, it compares the optimization results differently. Therefore, the execution time of this step is also measured to evaluate efficiency of TCE+ for detecting duplicated mutants. The results are presented in Table $\mathbf{1 3 .}$ 
Table 13. Execution Time of TCE+ for Detecting Equivalent and Duplicated Mutants

\begin{tabular}{|c|c|c|c|c|c|c|}
\hline & \multicolumn{6}{|c|}{ Execution Time (sec.) } \\
\cline { 2 - 7 } Program & Compile & Optimization & $\begin{array}{c}\text { Comparison for } \\
\text { Detecting } \\
\text { Equivalent Mu- } \\
\text { tants }\end{array}$ & $\begin{array}{c}\text { Comparison } \\
\text { for Detecting } \\
\text { Duplicated } \\
\text { Mutants }\end{array}$ & $\begin{array}{c}\text { Total for De- } \\
\text { tecting Equiva- } \\
\text { lent Mutants }\end{array}$ & $\begin{array}{c}\text { Total for } \\
\text { Detecting } \\
\text { Duplicated } \\
\text { Mutants }\end{array}$ \\
\hline P1 & 36 & 68 & 1 & 1 & 105 & 105 \\
\hline P2 & 57 & 124 & 3 & 1 & 184 & 182 \\
\hline P3 & 137 & 289 & 6 & 3 & 432 & 429 \\
\hline P4 & 101 & 188 & 5 & 2 & 294 & 291 \\
\hline P5 & 235 & 617 & 12 & 5 & 864 & 857 \\
\hline
\end{tabular}

As shown in Table 13, the execution times of detecting equivalent mutants and duplicated mutants do not differ noticeably, and they are about 1 second per mutant. Therefore, to answer RQ2, we conclude that TCE+ can be considered as an efficient method. Further, the comparison times, both for equivalent and duplicated mutants, are negligible. However, the optimization time is about 2-3 times the compile time. It is worth noting that the compile time is an inherent overhead of mutation testing, since in mutation testing, each mutant should be compiled and executed against the test cases. Therefore, the overhead imposed by TCE+ is the optimization time. Considering the fact that TCE+ can effectively detect equivalent and duplicate mutants, and these mutants do not need to be executed over the test cases, it means that TCE+ reduces the cost of mutation testing by reducing the number of mutants that need to be run and specially by removing the mutants that due to their equivalence, can waste the time of the test case designers. Hence, we believe the overhead of optimization time which involves CPU cycles can be considered as acceptable by the reduction it provides in required human effort. Consequently, we conclude that TCE+ is cost effective.

\section{Conclusion}

In this paper, the performance of TCE technique for detecting equivalent mutants in Java programs is evaluated. As the experimental evaluations have demonstrated, TCE has not detected any equivalent mutant in the subject programs and hence it cannot be considered to effective. To address this problem, current paper has proposed the TCE+ technique which extends TCE by utilizing an obfuscator like ProGuard, capable of performing some optimizations on Java programs.

The experimental evaluations show that while there are mutation operators like ROR for which TCE+ performance is weak, there are also operators like AOIS that TCE+ is able to find all of its equivalent mutants. Considering the contribution of each operator to the number of equivalent mutants of a typical program, TCE+ can be considered to be an effective and efficient method for detecting both equivalent and duplicated mutants for Java programs. 
TCE+: an Extension of the TCE Method for Detecting Equivalent Mutants in Java Programs

Current paper has investigated performance of TCE+ on small programs. Hence, it is required to perform similar experiments on larger Java programs to see how the performance of TCE+ changes as the program size increases. A challenge in this regard is preparation of the golden standard, since for large programs, the number of mutants is noticeable and it needs considerable effort to build a reliable golden standard. This is a main direction of our future work. Further, more precise analysis of the behavior of TCE+ on different mutation operators is an important job that we have scheduled for our future works. The results of such analysis will provide insights on possible improvements on ProGuard from the specific point of view of equivalent mutant detection.

\section{$5 \quad$ References}

1. Jia, Yue, and Mark Harman. "An analysis and survey of the development of mutation testing." IEEE transactions on software engineering 37.5 (2011): 649-678.

2. Budd, Timothy A., and Dana Angluin. "Two notions of correctness and their relation to testing." Acta Informatica 18.1 (1982): 31-45.

3. Offutt, A. Jefferson, and Jie Pan. "Automatically detecting equivalent mutants and infeasible paths." Software testing, verification and reliability 7.3 (1997): 165-192.

4. Madeyski, Lech, et al. "Overcoming the equivalent mutant problem: A systematic literature review and a comparative experiment of second order mutation." IEEE Transactions on Software Engineering 40.1 (2014): 23-42.

5. Schuler, David, and Andreas Zeller. "Covering and uncovering equivalent mutants." Software Testing, Verification and Reliability 23.5 (2013): 353-374.

6. Papadakis, Mike, and Yves Le Traon. "Mutation testing strategies using mutant classification." Proceedings of the 28th Annual ACM Symposium on Applied Computing. ACM, 2013.

7. Adamopoulos, Konstantinos, Mark Harman, and Robert M. Hierons. "How to overcome the equivalent mutant problem and achieve tailored selective mutation using co-evolution." Genetic and evolutionary computation conference. Springer Berlin Heidelberg, 2004.

8. Kintis, Marinos, and Nicos Malevris. "Using data flow patterns for equivalent mutant detection." Software Testing, Verification and Validation Workshops (ICSTW), 2014 IEEE Seventh International Conference on. IEEE, 2014.

9. Jia, Yue, and Mark Harman. "Higher order mutation testing." Information and Software Technology 51.10 (2009): 1379-1393.

10.Hierons, Rob, Mark Harman, and Sebastian Danicic. "Using program slicing to assist in the detection of equivalent mutants." Software Testing, Verification and Reliability 9.4 (1999): 233-262.

11. Schuler, David, and Andreas Zeller. "(Un-) Covering Equivalent Mutants." 2010 Third International Conference on Software Testing, Verification and Validation. IEEE, 2010.

12.Papadakis, Mike, et al. "Trivial compiler equivalence: A large scale empirical study of a simple, fast and effective equivalent mutant detection technique." 2015 IEEE/ACM 37th IEEE International Conference on Software Engineering. Vol. 1. IEEE, 2015.

13.Kintis, Marinos, and Nicos Malevris. "Identifying more equivalent mutants via code similarity." 2013 20th Asia-Pacific Software Engineering Conference. Vol. 1. IEEE, 2013.

14.Vincenzi, Auri Marcelo Rizzo, et al. "Bayesian-learning based guidelines to determine equivalent mutants." International Journal of Software Engineering and Knowledge Engineering 12.06 (2002): 675-689. 
15.Nica, Simona, and Franz Wotawa. "Using constraints for equivalent mutant detection." arXiv preprint arXiv:1207.2234 (2012).

16.Just, René, Michael D. Ernst, and Gordon Fraser. "Using state infection conditions to detect equivalent mutants and speed up mutation analysis." arXiv preprint arXiv:1303.2784 (2013).

17.Rojas, José Miguel, and Gordon Fraser. "Code Defenders: A Mutation Testing Game." The 11th International Workshop on Mutation Analysis. IEEE. 2015.

18.Kintis, Marinos, and Nicos Malevris. "MEDIC: A static analysis framework for equivalent mutant identification." Information and Software Technology 68 (2015): 1-17.

19. Yao, Xiangjuan, Mark Harman, and Yue Jia. "A study of equivalent and stubborn mutation operators using human analysis of equivalence." Proceedings of the 36th International Conference on Software Engineering. ACM, 2014.

20.Harman, Mark, Yue Jia, and William B. Langdon. "A manifesto for higher order mutation testing." Software Testing, Verification, and Validation Workshops (ICSTW), 2010 Third International Conference on. IEEE, 2010.

21.Nguyen, Quang Vu, and Lech Madeyski. "Searching for strongly subsuming higher order mutants by applying multi-objective optimization algorithm." Advanced Computational Methods for Knowledge Engineering. Springer International Publishing, 2015. 391-402.

22.Omar, Elmahdi, Sudipto Ghosh, and Darrell Whitley. "Constructing subtle higher order mutants for Java and AspectJ programs." 2013 IEEE 24th International Symposium on Software Reliability Engineering (ISSRE). IEEE, 2013.

23.Ma, Yu-Seung, Jeff Offutt, and Yong-Rae Kwon. "MuJava: a mutation system for Java." Proceedings of the 28th international conference on Software engineering. ACM, 2006.

24.Just, René, et al. "Are mutants a valid substitute for real faults in software testing?." Proceedings of the 22nd ACM SIGSOFT International Symposium on Foundations of Software Engineering. ACM, 2014.

25.Weitao, Wang, and Hirohide Haga. "Improvement of Equivalent Mutant Detection Using Loop Count Restriction." The International Conference on Software Engineering, Mobile Computing and Media Informatics (SEMCMI2015). 2015.

26.Diehl, Stephan. "A formal introduction to the compilation of Java." Software-Practice and Experience 28.3 (1998): 297-327. 